\title{
Contemporary Procedural Trends of Watchman Percutaneous Left Atrial Appendage Occlusion in the United States
}

\author{
Muhammad Bilal Munir ${ }^{1}$, Muhammad Khan ${ }^{2}$, Douglas Darden ${ }^{3}$, Deepak Kumar \\ Pasupula ${ }^{4}$, Sudarshan Balla ${ }^{2}$, Frederick $\mathrm{Han}^{3}$, Ryan Reeves ${ }^{5}$, and Jonathan $\mathrm{Hsu}^{6}$ \\ ${ }^{1}$ West Virginia University School of Medicine \\ ${ }^{2}$ West Virginia University Hospitals \\ ${ }^{3}$ University of California San Diego Health System \\ ${ }^{4}$ UPMC Passavant \\ ${ }^{5} \mathrm{UCSD}$ \\ ${ }^{6}$ University of California, San Diego
}

September 16, 2020

\begin{abstract}
Objective: To determine trends in real-world utilization and in-hospital adverse events from Watchman implantation since its approval by the Food and Drug Administration in 2015. Background: The risk of embolic stroke caused by atrial fibrillation is reduced by oral anticoagulants, but not all patients can tolerate long-term anticoagulation. Left atrial appendage occlusion with the Watchman device has emerged as an alternative therapy. Methods: This was a retrospective cohort study utilizing data from National Inpatient Sample for calendar years 2015-2017. The outcomes assessed in this study were associated complications, inhospital mortality, and resource utilization trends after Watchman implantation. Trends analysis were performed using analysis of variance. Multivariable adjusted logistic regression analysis was performed to determine predictors of mortality. Results: A total of 17,700 patients underwent Watchman implantation during the study period. There was a significantly increased trend in the number of Watchman procedures performed over the study years (from 1,195 in 2015 to 11,165 devices in 2017, p < 0.01). A significant decline in the rate of complications (from $26.4 \%$ in 2015 to $7.9 \%$ in $2017, \mathrm{p}<0.01$ ) and inpatient mortality (from $1.3 \%$ in 2015 to $0.1 \%$ in $2017, \mathrm{p}<0.01)$ were noted. Predictors of in-hospital mortality included a higher CHA2DS2-VASc score (OR 2.61 per 1-point increase, 95\% CI 1.91-3.57), chronic blood loss anemia (OR 3.63, 95\% CI 1.37-9.61) and coagulopathy (OR 4.90, 95\% CI 2.32-10.35). Conclusion: In contemporary United States clinical practice, Watchman utilization has increased significantly since approval in 2015, while complications and in-patient mortality have declined.
\end{abstract}

\section{Contemporary Procedural Trends of Watchman Percutaneous Left Atrial Appendage Occlu- sion in the United States}

Muhammad Bilal Munir, MD ${ }^{1,2^{*}}$, Muhammad Zia Khan, $\mathrm{MD}^{2 *}$, Douglas Darden, $\mathrm{MD}^{1}$, Deepak Kumar Pasupula, MD ${ }^{3}$, Sudarshan Balla, MD², Frederick T. Han, MD ${ }^{1}$, Ryan Reeves, MD ${ }^{1}$, Jonathan C. Hsu, MD, MAS $^{1}$

1. Section of Electrophysiology, Division of Cardiology, University of California San Diego, La Jolla, CA, USA

2. Division of Cardiovascular Medicine, West Virginia University Heart $\&$ Vascular Institute, Morgantown, WV, USA

3. Division of Cardiology, Heart and Vascular Institute, University of Pittsburgh Medical Center, Pittsburgh, PA, USA 


\section{Corresponding author:}

Jonathan C. Hsu, MD, MAS

Associate Professor of Medicine

University of California San Diego

9452 Medical Center Dr., MC7411, La Jolla, CA 92037

Email: Jonathan.Hsu@ucsd.edu

Conflicts:

Dr. Hsu reports receiving honoraria from Medtronic, Abbott, Boston Scientific, Biotronik, Janssen Pharmaceuticals, Bristol-Myers Squibb, Altathera Pharmaceuticals, Zoll Medical, and Biosense-Webster, research grants from Biotronik and Biosense-Webster, and has equity interest in Acutus Medical and Vektor Medical.

Word count: 2683

*Both Drs. Munir and Khan contributed equally to the manuscript.

\section{ABSTRACT:}

\section{Objective:}

To determine trends in real-world utilization and in-hospital adverse events from Watchman implantation since its approval by the Food and Drug Administration in 2015.

\section{Background:}

The risk of embolic stroke caused by atrial fibrillation is reduced by oral anticoagulants, but not all patients can tolerate long-term anticoagulation. Left atrial appendage occlusion with the Watchman device has emerged as an alternative therapy.

\section{Methods:}

This was a retrospective cohort study utilizing data from National Inpatient Sample for calendar years 20152017. The outcomes assessed in this study were associated complications, in-hospital mortality, and resource utilization trends after Watchman implantation. Trends analysis were performed using analysis of variance. Multivariable adjusted logistic regression analysis was performed to determine predictors of mortality.

\section{Results:}

A total of 17,700 patients underwent Watchman implantation during the study period. There was a significantly increased trend in the number of Watchman procedures performed over the study years (from 1,195 in 2015 to 11,165 devices in 2017, $\mathrm{p}<0.01$ ). A significant decline in the rate of complications (from $26.4 \%$ in 2015 to $7.9 \%$ in 2017, p < 0.01) and inpatient mortality (from $1.3 \%$ in 2015 to $0.1 \%$ in $2017, \mathrm{p}<0.01$ ) were noted. Predictors of in-hospital mortality included a higher CHA2DS2-VASc score (OR 2.61 per 1-point increase, 95\% CI 1.91-3.57), chronic blood loss anemia (OR 3.63, 95\% CI 1.37-9.61) and coagulopathy (OR $4.90,95 \%$ CI $2.32-10.35)$.

\section{Conclusion:}

In contemporary United States clinical practice, Watchman utilization has increased significantly since approval in 2015, while complications and in-patient mortality have declined.

Key words: Watchman; national trends; complications; mortality

\section{ABBREVIATIONS LIST:}

$\mathrm{AF}=$ atrial fibrillation 
LAA $=$ left atrial appendage

DOACs $=$ direct acting oral anti-coagulants

FDA $=$ Food and Drug Administration

LOS $=$ length of stay

$\mathrm{OR}=$ odds ratio

NIS $=$ National Inpatient Sample

ICD-9-CM = International Classification of Diseases, 9th Revision, Clinical Modification

ICD-10-CM = International Classification of Diseases, 10th Revision, Clinical Modification

\section{INTRODUCTION:}

Atrial fibrillation (AF) is the most commonly encountered sustained cardiac arrhythmia in clinical practice and responsible for more than $20 \%$ of all embolic strokes $(1,2)$. AF-associated strokes tend to have worse morbidity and mortality when compared to strokes not related to $\mathrm{AF}(3,4)$. The left atrial appendage (LAA) is the location for thrombus formation in more than $90 \%$ of patients with non-valvular AF (5). Coumadin and direct oral anti-coagulants (DOACs) are the gold-standard therapy for reducing stroke risk in AF patients with risk factors for stroke. However, their utilization is often limited by lack of patient compliance and adverse effects $(6,7,8)$. Left atrial appendage occlusion (LAAO) using an endocardial Watchman device has shown promising results in mitigating stroke risk when utilized in selected AF patients (9). The landmark PROTECT (Percutaneous closure of the left atrial appendage versus warfarin therapy for prevention of stroke in patients with atrial fibrillation) trial showed the Watchman device to be non-inferior to coumadin in terms of the primary efficacy end-point of stroke, systemic embolism and cardiovascular/unexplained death (10). Subsequently, the PREVAIL (Prospective randomized evaluation of the Watchman left atrial appendage closure device in patients with atrial fibrillation versus long-term warfarin therapy) trial confirmed these results and also showed a reduced rate of short-term complications (11). The results of these two trials eventually led to Food and Drug Administration (FDA) approval of the Watchman device in March of 2015. Since FDA approval of the Watchman device, there has been limited real-world data on trends in utilization, complications and in-hospital mortality from the procedure in contemporary practice. The aim of the present study is to assess these parameters from a comprehensive, national United States (US) population database.

\section{METHODS:}

Study data:

For the purpose of the current analysis, data were derived from the National Inpatient Sample (NIS) for calendar years 2015-2017. The NIS is made possible by a Federal-State-Industry partnership sponsored by the Agency for Healthcare Research and Quality (AHRQ). The NIS is derived from all States for national estimates of healthcare utilization, costs and outcomes (12). NIS data are compiled annually and therefore the data can be used for analyses of disease trends over time. The NIS approximates $20 \%$ of all discharges from all US non-Federal hospitals and provides discharge weights that are used for computation of national estimates. Institutional Review Board approval and informed consents were not required for this study given the de-identified nature of the NIS dataset and public availability.

Study population and study design:

We analyzed NIS data from January 2015 to December 2017. The study population was selected by using the International Classification of Diseases, 9th Revision, Clinical Modification (ICD-9-CM) and International Classification of Diseases, 10th Revision, Clinical Modification (ICD-10-CM) codes. We selected patients 18 years of age and above for the purpose of our study. Age was further divided into three groups, $<65,65-74$ and [?]75. Patients implanted with a WATCHMAN were identified by ICD-9 code of 37.90 and ICD-10 code of 02L73DK. Baseline characteristics and key complications were identified (ICD codes for complications 
provided in supplement), as previously described (13). Hospital outcomes including in-patient mortality, discharge disposition, length of stay (LOS) and cost of hospitalization (inflation adjusted) were derived. For the computation of hospitalization costs, cost-to-charge ratio files from NIS were utilized.

Complication rate trends were analyzed over the study years. The primary outcome of the study was the prevalence of Watchman implantation over our study period. Additional outcomes analyzed including associated complications, in-hospital mortality and resource utilization (including LOS, cost of stay and discharge disposition to home, short term care, long-term care or home with institutional care or home health).

Statistical analysis:

Descriptive statistics are presented as frequencies with percentages for categorical variables and as means with standard deviations for continuous variables. Baseline characteristics were compared using a Pearson?2 test and Fisher's exact test for categorical variables and independent samples t-test for continuous variables. Trends analysis was performed using Analysis of variance (ANOVA). Linear regression was used to predict trends over calendar years. Logistic regression was performed to estimate odds ratios (ORs) with $95 \%$ confidence intervals (CIs) to determine predictors for mortality. Initially, a binomial logistic regression model was used to identify variables from demographic data (Table 1) that were significantly associated with patient mortality ( $\mathrm{P}$ value $; 0.10$ ). These variables were then subsequently utilized in a multivariable logistic regression model to identify statistically significant predictors of mortality. In the final model, P-value of $<0.05$ was used as cutoff for stepwise forward entry for logistic regression. A type I error rate of $<0.05$ was considered statistically significant. All statistical analyses were performed using statistical package for social science (SPSS) version 26 (IBM Corp). Discharge weights provided by NIS were used for computation of national estimates. All analyses were done on a weighted sample.

\section{RESULTS:}

A total of 17,700 patients underwent Watchman implantation from January 2015 to December 2017. The mean age of patients implanted was 75.6 ( $\mathrm{SD} \pm 8.2$ ) years. The mean age increased over the study years (74.2 years in year 2015 vs. 75.8 years in year $2017, \mathrm{P}<0.01)$. Overall, women constituted $40.1 \%(\mathrm{n}=7,095)$ of the study cohort, and the majority of patients $86.0 \%(\mathrm{n}=14,650)$ were White. Baseline characteristics of the study population are shown in table 1. Between 2015 and 2017, patients undergoing LAAO in later calendar years were on average older, more commonly female, and more commonly electively admitted for the procedure.

Overall, there was a significant increase in the number of Watchman LAAO procedures in the US (from 1,195 device implants in year 2015 to 11,165 device implants in year 2017, p < 0.01, figure 1). Peri-procedural complications associated with Watchman implantation are depicted in table 2 . There was a significant decrease in the rate of complications over the study period $(26.4 \%$ in year 2015 vs. $7.9 \%$ in year $2017, \mathrm{p}<0.01)$. The largest decrease in complications over the study years occurred with cardiovascular and neurological complications, with a decreased rate of any cardiovascular complication from $13.8 \%$ in year 2015 to $4.7 \%$ in year $2017(\mathrm{p}<0.01)$, while the rate of any neurological complication decreased from $7.9 \%$ in year 2015 to just $0.9 \%$ in year $2017(\mathrm{p}<0.01)$. There were very low rates of device related thrombus at discharge or device embolization during the study period ( $<10$ patients; $<0.1 \%$ ). Overall, in-hospital mortality was low at $0.3 \%$ ( $\mathrm{n}=45$ patients), and mortality decreased each year from $1.3 \%$ in 2015 to $0.1 \%$ in 2017 (figure 2).

Multivariable adjusted predictors of mortality for patients undergoing Watchman implantation are shown in figure 5. A higher CHA2DS2-VASc score (OR 2.61 for each 1 point increase in score [95\% CI 1.91-3.57], $\mathrm{p}<0.01$ ), chronic blood loss anemia (OR 3.63 [95\% CI 1.37-9.61], $\mathrm{p}<0.01$ ) and coagulopathy (OR 4.90 [95\% CI 2.32-10.35], $\mathrm{p}<0.01$ ) were associated with a higher mortality, whereas a more recent calendar year of implant (OR 0.28 per year increase [95\% CI 0.19-0.43], $\mathrm{p}<0.01$ ) and history of hypertension (OR 0.28 [95\% CI 0.14-0.56], $\mathrm{p}<0.01$ ) were associated with a lower odds of mortality at discharge.

As seen in Table 3, the majority of patients were discharged home or with home healthcare. Both LOS and 
cost of hospitalization demonstrated a declining trend over the study period (Table 3 and figures 3 and 4).

\section{DISCUSSION:}

The main findings of our current investigation are: (1) Over the study period from 2015-2017, there has been a significant increase in the number of Watchman device implantation procedures in the United States (from 1,195 in year 2015 to 11,165 devices in year 2017, $\mathrm{p}<0.01$ ). (2) There has been a decline in the rate of complications over the study period primarily driven by lower rates of cardiovascular and neurological complications. (3) Overall mortality continues to be low during the study period, with an even lower trend towards reduced mortality over the study years $(1.3 \%$ in year 2015 vs. $0.1 \%$ in year $2017, \mathrm{p}<0.01)$. (4) The total length of stay and hospitalization costs after Watchman implantation declined over the study period.

Percutaneous LAAO with the Watchman device provides a viable alternative to oral anticoagulation in select patients based on randomized trials that have shown efficacy and safety of utilizing this approach for stroke risk reduction $(10,11)$. The FDA approved the device for commercial use in United States in March of 2015 and the Watchman implant procedure currently carries a class IIb recommendation in patients with nonvalvular AF at risk for stroke per the latest American College of Cardiology guidelines (14). Our analysis of a contemporary, real-world, national database sampling US practice since FDA approval of Watchman showed consistent increased utilization of device procedures suggesting gradual assimilation of this device implantation procedure in clinical practice. Additionally, our analysis also showed that complications and in-patient mortality associated with implantation of Watchman devices continued to show a downward trend.

Our study showed a significant decline in the overall complication rate over the study period (26.4\% in year 2015 vs. $7.9 \%$ in year $2017, \mathrm{p}<0.01)$. This downtrend was primarily driven by a reduction in cardiovascular and neurological complications over the study period. Cardiac perforation complications including cardiac tamponade were encountered in the landmark PROTECT trial where its prevalence was approximately $4.3 \%$ (10). Subsequently, with improved operator experience and better implant techniques, the incidence of this complication was lowered to $1.9 \%$ in the PREVAIL trial, 1.4\% in CAP (Continued Access to PROTECT), $1.9 \%$ in CAP2 (Continued Access to PREVAIL), and $0.3 \%$ in EWOLUTION registries $(11,15,16)$. The overall rate of cardiac tamponade in the current study of contemporary Watchman patients was $0.8 \%$. This rate is similar to post-FDA approval study led by Reddy et al. in which the authors reported a nearly similar rate of cardiac tamponade at 1\%; still higher than the European EWOLUTION registry which reported cardiac tamponade rate of $0.3 \%$ (17). It is also similar to a recently published contemporary registry of Watchman implantations from National Cardiovascular Data Registry which analyzed nearly 38,000 patients (18). It may be expected that with more widespread availability of the Watchman device in US practice that the rate of cardiac tamponade may continue to decline over the coming years. The rate of ischemic stroke/transient ischemic attack (TIA) was $1 \%$ in our study cohort with most cases reported in 2015 and a significant downtrend since that time $(0.7 \%$ in year 2016 and $0.4 \%$ in year 2017, p < 0.01). Vuddanda et al. have shown nearly similar ischemic stroke/TIA rates of about $0.5 \%$ when analyzing Watchman implants from year 2016 (13). These strokes are presumed to be due to inadvertent air or clot embolization from the trans-septal sheath and enhanced physician training should continue to mitigate this risk.

The current study showed a downward trend in in-hospital mortality suggesting improved safety with the device with more operator experience (from $1.3 \%$ in year 2015 to $0.1 \%$ in year $2017, \mathrm{p}<0.01$ ). Improvement in mortality rate trends over time seen in the current study of real-world patients was also seen in previous clinical studies, including the CAP2 registry which supplemented the PREVAIL trial and was designed to continue long-term accrual of data, and showed a mortality rate of $0.2 \%$ within 7 days of Watchman implant (15). A subsequent study utilizing National Inpatient Sample database by Vuddanda showed a mortality rate of $0.3 \%$ at discharge for combined endocardial and epicardial based approaches for LAA occlusion (13). In another post-FDA approval analysis of more than 3,800 patients undergoing Watchman implantation from March, 2015 to May, 2016, Reddy et al. demonstrated procedure related mortality of $0.078 \%$ (17). The prospective EWOLUTION registry that enrolled more than 1,000 consecutive patients undergoing Watchman implantation outside the US also showed low procedure related mortality of $0.1 \%$ (16). These earlier studies along with our more contemporary data suggest that overall implantation of the 
Watchman device is associated with low absolute rates of mortality that is decreasing over time.

\section{Limitations:}

Our study has the following key limitations: (1) The NIS is an administrative claims-based database that utilized ICD codes which may be prone to errors and could introduce information bias due to outcome misclassification. The hard clinical end points, however, are less subjected to error. Additionally, AHRQ quality control measures are routinely instituted that guarantee data integrity (12). Additionally, the ICD-9 code utilized in this study was not specific to the Watchman device and could be referred for any LAA occlusion procedure. Due to the limited magnitude of other research studies of endocardial devices and any epicardial LAA occlusion procedures performed in US during the study period (13), we believe that application of this code for the purpose of our study was able to mostly characterize Watchman implants. (2) The NIS only captures inpatient admissions and does not provide any information on outpatient encounters. This limitation may result in selection bias; however, our data is well representative of national utilization of Watchman devices performed during in-patient settings; in fact since inpatient hospitalization is often required for reimbursement for the procedure, our results may be more indicative of widespread practice (19). (3) The NIS censors data gathering at discharge so long-term outcomes could not be ascertained from the present dataset. (4) Specific data on potential confounders including medications, as well as operator and intra-procedural characteristics could not be examined from the NIS.

\section{Conclusion:}

In conclusion, in this large, nationally representative sample of a United States database, there has been a significant increase in the use of Watchman devices since FDA approval in 2015. Between 2015 and 2017, a significantly reduced rate of procedural complications and in-hospital mortality was noted, which appeared primarily driven by reduction in cardiovascular and neurological complications. In later years, length of stay shortened and costs of hospitalization for the procedure decreased.

\section{Perspectives:}

\section{WHAT IS KNOWN:}

Watchman device has emerged as an alternative treatment for the management of stroke risk in AF patients who cannot tolerate oral anti-coagulation. Randomized trials have demonstrated non-inferiority of the Watchman device in terms of both efficacy and safety when compared to coumadin.

\section{WHAT IS NEW:}

In real-world US practice, increase in utilization of Watchman device was noted since its approval by FDA in 2015. Additionally, the rate of in-patient complications and mortality associated with Watchman implantation declined from years 2015 to 2017.

\section{WHAT IS NEXT:}

Further research is needed to characterize AF patients who are most likely to benefit from the Watchman device.

\section{Table and Figure Legends:}

Table 1: Baseline characteristics of the study population of patients undergoing Watchman device implantation, stratified by year of implant from years 2015 to 2017

Table 2: Complications in patients undergoing Watchman implantation

Table 3: Hospital outcomes and resource utilization of the study cohort

Figure 1: Number of Watchman procedures over the study years

Figure 2: Trends in Mortality after Watchman implant from years 2015 to 2017 
Figure 3: Mean length of stay during Watchman implantation admissions from years 2015 to 2017

Figure 4: Adjusted mean cost of stay during Watchman implantation admissions from years 2015 to 2017

Figure 5: Predictors of in-hospital mortality associated with Watchman implantation from years 2015 to 2017

\section{Supplemental Data:}

Supplementary 1: ICD-9 and ICD-10 codes used for the study cohort and variables entered in logistic regression model

\section{References:}

1. Mozaffarian D, Benjamin EJ, Go AS, Arnett DK, Blaha MJ, Cushman M, et al. Heart Disease and Stroke Statistics-2016 Update: A Report From the American Heart Association. Circulation. 2016;133:e38-360

2. Chugh SS, Havmoeller R, Narayanan K, Singh D, Rienstra M, Benjamin EJ, et al. Worldwide epidemiology of atrial fibrillation: a Global Burden of Disease 2010 Study. Circulation. 2014;129:837-47

3. Saposnik G, Gladstone D, Raptis R, Hart RG. Atrial fibrillation in ischemic stroke: predicting response to thrombolysis and clinical outcomes. Stroke. 2013;44:99-104

4. Seet RC, Zhang Y, Wijdicks EF, Rabinstein AA. Relationship between chronic atrial fibrillation and worse outcomes in stroke patients after intravenous thrombolysis. Arch Neurol. 2011;68:1454-1458

5. Alkhouli M, Noseworthy PA, Rihal CS, Holmes DR Jr. Stroke Prevention in Nonvalvular Atrial Fibrillation: A Stakeholder Perspective. J Am Coll Cardiol. 2018;71:2790-2801

6. Hayden DT, Hannon N, Callaly E, Ní Chróinín D, Horgan G, Kyne L, et al. Rates and Determinants of 5-Year Outcomes After Atrial Fibrillation-Related Stroke: A Population Study. Stroke. 2015;46:3488-93

7. Oldgren J, Healey JS, Ezekowitz M, Commerford P, Avezum A, Pais P, et al. Variations in cause and management of atrial fibrillation in a prospective registry of 15,400 emergency department patients in 46 countries: the RE-LY Atrial Fibrillation Registry. Circulation. 2014;129:1568-76

8. Hsu JC, Maddox TM, Kennedy KF, Katz DF, Marzec LN, Lubitz SA, et al. Oral Anticoagulant Therapy Prescription in Patients With Atrial Fibrillation Across the Spectrum of Stroke Risk: Insights From the NCDR PINNACLE Registry. JAMA Cardiol. 2016;1:55-62

9. Holmes DR Jr, Alkhouli M, Reddy V. Left Atrial Appendage Occlusion for The Unmet Clinical Needs of Stroke Prevention in Nonvalvular Atrial Fibrillation. Mayo Clin Proc. 2019;94:864-874

10. Holmes DR, Reddy VY, Turi ZG, Doshi SK, Sievert H, Buchbinder M, et al. Percutaneous closure of the left atrial appendage versus warfarin therapy for prevention of stroke in patients with atrial fibrillation: a randomised non-inferiority trial. Lancet. 2009;374:534-42

11. Holmes DR Jr, Kar S, Price MJ, Whisenant B, Sievert H, Doshi SK, et al. Prospective randomized evaluation of the Watchman Left Atrial Appendage Closure device in patients with atrial fibrillation versus long-term warfarin therapy: the PREVAIL trial. J Am Coll Cardiol. 2014;64:1-12

12. Agency for Healthcare Research and Quality. Overview of the national inpatient sample (NIS). Rockville: AHRQ. https://www.hcup-us.ahrq.gov/nisoverview.jsp. Accessed on January 21, 2020

13. Vuddanda VLK, Turagam MK, Umale NA, Shah Z, Lakkireddy DR, Bartus K, et al. Incidence and causes of in-hospital outcomes and 30-day readmissions after percutaneous left atrial appendage closure: A US nationwide retrospective cohort study using claims data. Heart Rhythm. 2019 Sep 17

14. January CT, Wann LS, Calkins H, Chen LY, Cigarroa JE, Cleveland JC Jr, et al. 2019 AHA/ACC/HRS focused update of the 2014 AHA/ACC/HRS guideline for the management of patients with atrial fibrillation: A Report of the American College of Cardiology/American Heart Association Task Force on Clinical Practice Guidelines and the Heart Rhythm Society. Heart Rhythm. 2019;16:e66-e93

15. Holmes DR Jr, Reddy VY, Gordon NT, Delurgio D, Doshi SK, Desai AJ, et al. Long-Term Safety and Efficacy in Continued Access Left Atrial Appendage Closure Registries. J Am Coll Cardiol. 2019;74:2878-2889

16. Boersma LV, Schmidt B, Betts TR, Sievert H, Tamburino C, Teiger E, et al. Implant success and 
safety of left atrial appendage closure with the WATCHMAN device: peri-procedural outcomes from the EWOLUTION registry. Eur Heart J. 2016;37:2465-74

17. Reddy VY, Gibson DN, Kar S, O'Neill W, Doshi SK, Horton RP, et al. Post-Approval U.S. Experience With Left Atrial Appendage Closure for Stroke Prevention in Atrial Fibrillation. J Am Coll Cardiol. 2017;69:253-261

18. Freeman JV, Varosy P, Price MJ, Slotwiner D, Kusumoto FM, Rammohan C, Kavinsky CJ, Turi ZG, Akar J, Koutras C, Curtis JP, Masoudi FA. The NCDR Left Atrial Appendage Occlusion Registry. J Am Coll Cardiol. 2020;75:1503-1518

19. Centers for Medicare and Medicaid Services Coverage with Evidence Development (Left Atrial Appendage Occlusion). https://www.cms.gov/Medicare/Coverage/Coverage-with-Evidence- Development/LAAC. Accessed on March 16, 2020

\section{Hosted file}

Table 1 Baseline characteristics.docx available at https://authorea.com/users/353564/ articles/481433-contemporary-procedural-trends-of-watchman-percutaneous-left-atrialappendage-occlusion-in-the-united-states

\section{Hosted file}

Table 2 Complications clean.docx available at https://authorea.com/users/353564/articles/ 481433-contemporary-procedural-trends-of-watchman-percutaneous-left-atrial-appendageocclusion-in-the-united-states

\section{Hosted file}

Table 3 Hospital outcomes.docx available at https://authorea.com/users/353564/articles/ 481433-contemporary-procedural-trends-of-watchman-percutaneous-left-atrial-appendageocclusion-in-the-united-states

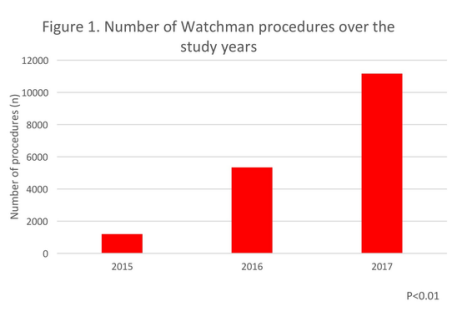




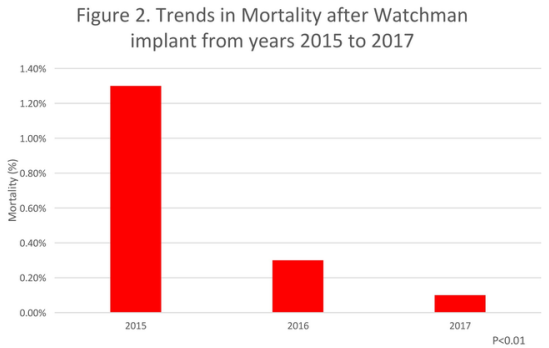

Figure 3. Mean length of stay during Watchman implantation admissions from years 2015 to

$$
2017
$$

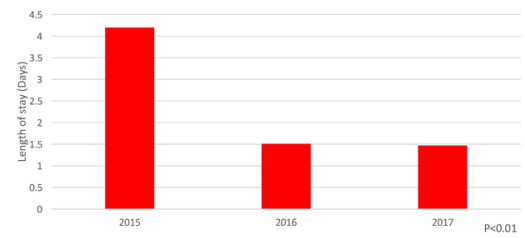

Figure 4. Adjusted mean cost of stay during Watchman implantation admissions from years 2015 to 2017

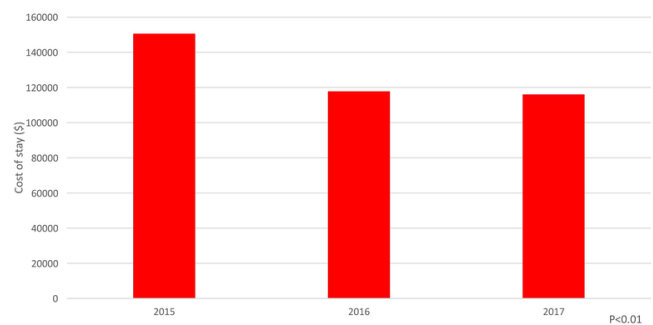




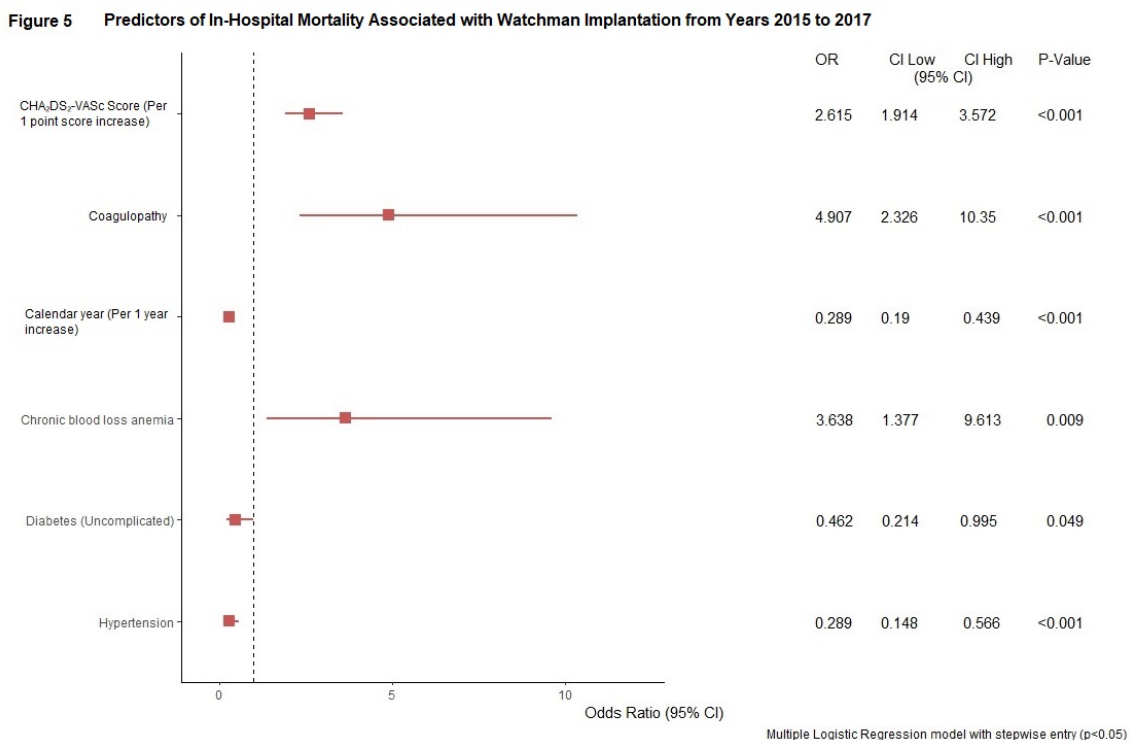

